\title{
The super star clusters of SBS 0335-052
}

\author{
Rodger I. Thompson ${ }^{1}$ \\ ${ }^{1}$ Steward Observatory, University of Arizona, Tucson, AZ 85721, USA \\ email: rthompson@as.arizona.edu
}

\begin{abstract}
WFPC2 continuum images of the low metallicity Blue Compact Dwarf Galaxy SBS 0335-052 revealed 6 intense point source that were deemed to be super star clusters. Subsequent observations with NICMOS in the infrared continuum and Paschen $\alpha$ reveals that only 2 of the sources have very young stars with the other point sources as clusters that may have ended their episodes of star formation. The optical images show a probable supernova created cavity to the north of the present clusters, while the currently active star forming clusters lie at the southern end of the cluster. This indicates a possible string of induced star formation propagating from the north to the south. The presence of a large number of $\mathrm{O}$ stars in a small volume raises the question of whether super star clusters might be the breeding grounds for intermediate mass black holes.
\end{abstract}

\section{Introduction}

SBS 0335-052 is a two component Blue Compact Dwarf galaxy that is embedded in a $2 \times 10^{9} L_{\odot}$ HI cloud (Pustilnik et al. 2001). The cloud is dark matter dominated according to Pustilnik et al. (2001) who find that the dynamical mass is 3 times the observed stellar and gas mass. It is the second lowest metallicity galaxy known with a metallicity approximately 1/40 solar (Izotov et al. 1992). The eastern component of the galaxy contains 6 unresolved objects in WFPC2 V and I images, termed super star clusters by Thuan, Izotov \& Lipovetsky (1997). Although not explicitly defined, we will take the term super star cluster to mean a cluster with a $N_{e}^{2} V$ value greater than 1000 equivalent $\mathrm{O} 7$ stars and confined to a volume of a few parsecs or less. In spite of its very low metallicity, SBS 0335-052 is the site of a strong mid-IR source found by ISO (Plante \& Sauvage 2002). The positional accuracy and spatial resolution of the ISO detection is not sufficient to identify the source with any of the optical point sources and the true source may in fact be hidden from optical view. The dynamical Hubble distance to SBS 0335-052 is only $53 \mathrm{Mpc}$ so that 1 arc second subtends $263 \mathrm{pc}$.

The exciting aspect of SBS0335-052 is that it appears to be a galaxy with very little previous star formation, as indicated by its low metallicity, that is now undergoing a burst of star formation, perhaps for the very first time. In this sense we can view star formation processes that must have been dominant in the early universe in great detail. We can watch the feedback processes at work that are one of the primary uncertainties in most theoretical work on galaxy formation. An intriguing aspect is a cavity in the northern extent of the cluster region, about 4 arc seconds, or $1 \mathrm{kpc}$ from the southern most sources. It appears to be a supernova cavity and may represent the initial location of star formation in SBS 0335-052. Recent Chandra results from Thuan et al. (2004) find x-ray emission associated with SBS 0335-052, with two principal components. The strongest is very near sources 1 and 2 (See Fig. 1), probably coincident with source 2 within the registration errors between the optical and x-ray images. The second source is at the northern cavity, strengthening its interpretation as a supernova cavity. 

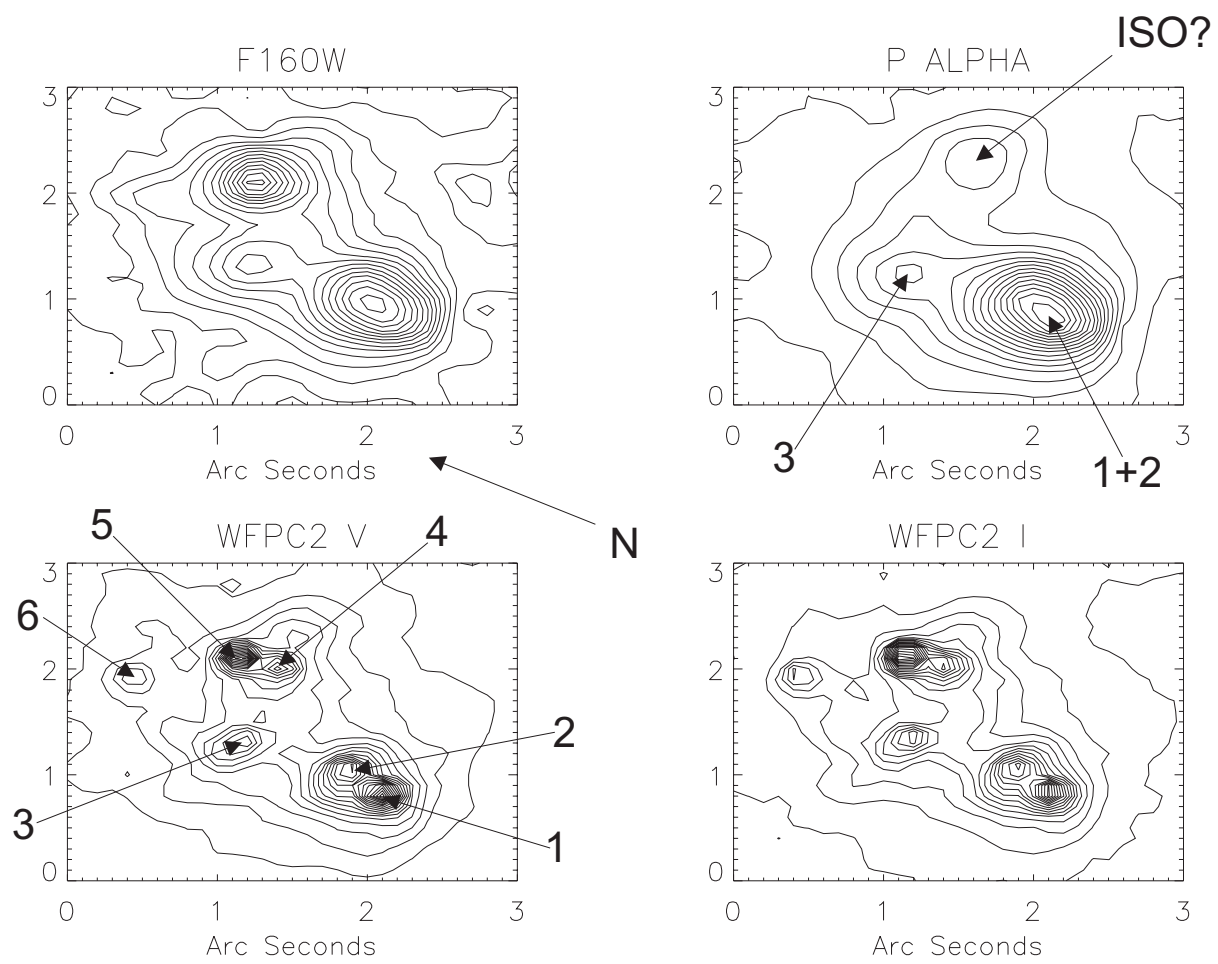

$\mathrm{N}$

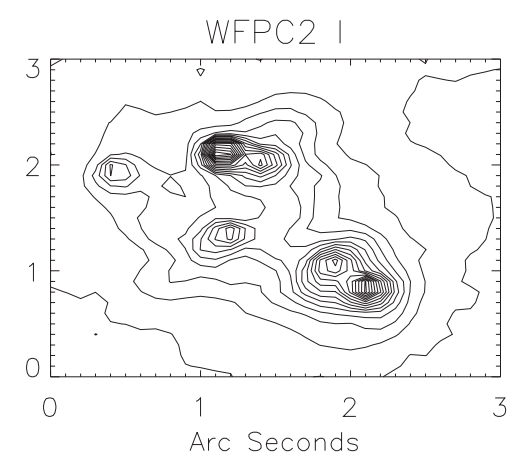

Figure 1. Contour plots of the super star cluster region in SBS0335-052. The labels in the WFPC2 V image contours indicate the 6 point sources discussed by Thuan, Izotov \& Lipovetsky (1997). The area labeled ISO in the Paschen $\alpha$ contours indicates the possible location of the ISO source.

One clue to the reason that SBS 0335-052 has finally initiated its first episode of major star formation is the presence of the large spiral galaxy NGC 1376 to the west. The radial velocities of the galaxies differ by only $150 \mathrm{~km} \mathrm{~s}^{-1}$ while the escape velocity at the projected separation of the two galaxies is $200 \mathrm{~km} \mathrm{~s}^{-1}$. The interaction between the two galaxies is the most likely trigger for the onset of star formation in SBS 0335-052. This may be the first lesson about early star formation. If the interaction time between a major galaxy and a smaller component is longer than the typical collapse time for star formation then significant star formation will occur in the minor component before the two components actually merge.

\section{Observations}

The new observations are HST NICMOS camera 3 images at 1.6 microns and in the hydrogen Paschen $\alpha$ line. The observations are part of the SINGS program of observations of galaxies with both HST and Spitzer, headed by Robert Kennicutt. The raw data was recovered from the HST archive and reduced with procedures developed for the NICMOS GTO data. The NICMOS and WFPC2 images were aligned using stars common to both of the images. The brightest star in both the NICMOS and WFPC2 has high proper motion with a significant change in position between the epochs of observation. It was discarded from the alignment procedure. Note that the redshift of SBS 0335-052 shifted the Paschen $\alpha$ line into the NICMOS narrow band continuum filter F190N leaving the usual line filter F187N as the continuum measurement. The contour plots of the 2 WFPC2 images and the NICMOS images are presented in Fig. 1 


$\begin{array}{lccc}\text { Source } & \mathrm{Pa} \alpha\left(\mathrm{ergs} \mathrm{s}^{-1}\right) & N_{e}^{2} V\left(\mathrm{~cm}^{-3}\right) & \text { Eq. O7 stars } \\ 1 \text { and } 2 & 4.7 \times 10^{39} & 8.7 \times 10^{64} & 1900 \\ 3 & 4.5 \times 10^{37} & 8.3 \times 10^{62} & 19 \\ 4 & \leqslant 5.0 \times 10^{37} & \leqslant 1.0 \times 10^{63} & \leqslant 23 \\ 5 & \leqslant 1.0 \times 10^{38} & \leqslant 2.0 \times 10^{63} & \leqslant 46 \\ 6 & \leqslant 1.0 \times 10^{38} & \leqslant 2.0 \times 10^{64} & \leqslant 46 \\ \text { ISO? } & 1.0 \times 10^{38} & 1.9 \times 10^{63} & 43 \\ \text { Total } & 1.2 \times 10^{40} & 2.1 \times 10^{65} & 4700\end{array}$

Table 1. Ionization properties of the clusters.

\section{Continuum and Line Images}

From Fig. 1 it is evident that the main features of the optical continuum are repeated at lower spatial resolution in the 1.6 micron continuum image. It is interesting that no new sources appear in the near infrared, indicating that there are no other sources of moderate extinction that would be invisible in the optical image, but starting to emerge in the infrared image. This was probably already indicated by the similarity between the $\mathrm{V}$ and I WFPC2 images. It should be noted, however, that the total 1.6 micron integration time was only 96 seconds since it was part of a general survey.

The Paschen $\alpha$ image, however, is quite different from the optical image. Sources 1 and 2 emerge as the only strong Paschen $\alpha$ sources with source 3 showing some weak emission. Sources 4, 5 and 6 have no emission peaks at their locations. There is diffuse Paschen $\alpha$ emission in the region that may be interstellar gas ionized by the stars in clusters 1 and 2, or reflected emission from dust in the intracluster medium. Integration of the total Paschen $\alpha$ flux indicates that the point sources contain only $1 / 2$ of the integrated flux, with the diffuse flux making up the other half. Table 1 lists the source fluxes and equivalent $N_{e}^{2} V$ values. The upper limits for sources 4,5 and 6 represent the diffuse flux in those regions.

If we take Paschen $\alpha$ emission as a measure of star formation, we can see a progression of star forming activity from north to south in SBS 0335-052. The oldest region is in the north, where supernova activity has cleared out the region and no new star formation is occurring. The intermediate age is represented by clusters 4,5 , and 6 where we only have upper limits on the star formation activity. Source 3 has limited star formation, which we might suspect as the last stage of active star formation. At the southern end of the complex are the very active star forming regions represented by sources 1 and 2 . If this scenario is true, then we see two different effects of feedback, both negative and positive. The northern supernova activity had negative feedback on that region, curtailing any future star formation by clearing out the region. That clearing process, on the other hand, may have produced positive feedback in the southern area by compressing the gas there and triggering a chain of star formation in existing over dense regions. Whether these regions would have formed stars eventually, without the extra push from the expanding supernova shell, is an open question. In any case, by the criterion established in the introduction, only sources 1 and 2 can currently be called super star clusters.

The region marked ISO? in Fig. 1 is a possible location of the dusty ISO source. It is a region with a peak of Paschen $\alpha$ emission without any equivalent peak in the 1.6 micron continuum. This signature is consistent with a hidden region of high star formation. In star formation regions with strong HII regions the equivalent width of Paschen $\alpha$ is very high. It is the most intense emission line longward of 1 micron. As such, it is the first 
feature in the near infrared to penetrate the dust extinction. It is not certain that this region is the origin of the observed mid-infrared emission, but it is a good candidate. If that region is the ISO source it does not follow the north to south progression of the star formation activity. On the other hand, it may lie significantly behind the optically observed clusters and represents star formation on the back side of the region. This would be consistent with its high extinction.

\section{Future Evolution}

The end point of the evolution of super star clusters is most likely represented by the globular clusters that we observe in our own and other galaxies. There is the possibility of another end point that is more exotic in nature. Super star clusters with several thousand O stars will produce several hundred black holes, generally thought to be the endpoint of evolution for stars with masses greater than $60 \mathrm{M}_{\odot}$. Typical super star clusters have dimensions of approximately $1 \mathrm{pc}$, however, core collapse of the more massive stars through equipartition of energy produces a very dense assemblage of the most massive objects. This is a possible mechanism for the creation of intermediate mass black holes, which are the first step toward the production of accretion driven AGN. Two new simulations (Gurkan, Freitag \& Rasio 2004, Portegies Zwart et al. 2004), support such a scenario. If this is the case then SBS 0335-052, along with being a local example of star formation at high redshift, might be giving us a close hand view of the creation of a quasar.

\section{Acknowledgements}

This work was done in collaboration with Marc Sauvage, Leslie Hunt, Rob Kennicutt and Chad Engelbracht. All errors should be attributed to the author and not the collaborators. This work contains data obtained from the NASA/ESA Hubble Space Telescope, which is operated by the Association of Universities for Research in Astronomy (AURA), Inc. under NASA contract NAS5-26555.

\section{References}

Gurkan, M. A., Freitag, M., \& Rasio, F. M. 2004, ApJ, 604, 632

Izotov, Y. I., Lipvetsky, V. A., Guseva, N. G., \& Kniazev, A. Y. 1992, In The Feedback of Chemical Evolution on the Stellar Content of Galaxies (eds. D. Alloin \& G. Stasinska). Paris: Obs. Paris, 138

Plante, S., \& Suvage, M. 2002, AJ, 124, 1995

Portegies Zwart, S., et al. 2004, (astro-ph/0402622)

Pustilnik 2001, AJ, 121, 1413

Thuan, T. X., Izotov, Y. I., \& Lipovetsky, V. A. 1997, ApJ, 477, 661

Thuan, T. X., Bauer, F. E., Papaderos, P., \& Izotov, Y. I. 2004, ApJ, 606, 213 